\title{
Cost-effectiveness analysis of malaria interventions using disability adjusted life years: a systematic review
}

\author{
Resign Gunda* (1) and Moses John Chimbari
}

\begin{abstract}
Background: Malaria continues to be a public health problem despite past and on-going control efforts. For sustenance of control efforts to achieve the malaria elimination goal, it is important that the most cost-effective interventions are employed. This paper reviews studies on cost-effectiveness of malaria interventions using disability-adjusted life years.

Methods: A review of literature was conducted through a literature search of international peer-reviewed journals as well as grey literature. Searches were conducted through Medline (PubMed), EMBASE and Google Scholar search engines. The searches included articles published in English for the period from 1996 to 2016. The inclusion criteria for the study were type of malaria intervention, year of publication and cost-effectiveness ratio in terms of cost per DALY averted. We included 40 studies which specifically used the DALY metric in cost-effectiveness analysis (CEA) of malaria interventions.
\end{abstract}

Results: The majority of the reviewed studies (75\%) were done using data from African settings with the majority of the interventions (60.0\%) targeting all age categories. Interventions included case treatment, prophylaxis, vector control, insecticide treated nets, early detection, environmental management, diagnosis and educational programmes. Sulfadoxine-pyrimethamine was the most common drug of choice in malaria prophylaxis, while artemisinin-based combination therapies were the most common drugs for case treatment. Based on guidelines for CEA, most interventions proved cost-effective in terms of cost per DALYs averted for each intervention.

Conclusion: The DALY metric is a useful tool for determining the cost-effectiveness of malaria interventions. This paper demonstrates the importance of CEA in informing decisions made by policy makers.

Keywords: Malaria, Disability-adjusted life years, Cost-effectiveness, Cost, Effectiveness

\section{Background}

Although the number of malaria cases are showing a declining trend, about 3.2 billion people remain at risk of malaria [1]. There were an estimated 214 million new cases of malaria and 438,000 deaths in 2015 alone, with approximately $80 \%$ of these deaths concentrated in just 15 countries, mainly in Africa [1]. The main challenges in the fight against malaria include in-effective national malaria control programmes, changes in population

*Correspondence: gundazr@yahoo.co.uk

School of Nursing and Public Health, College of Health Sciences,

University of KwaZulu-Natal, Howard Campus, Durban 4001, South Africa distribution and population growth, changes in land use, resistance to anti-malarial drugs, insecticide resistance, poor health infrastructure as well as climate change and climate variability $[1,2]$.

Cost-effectiveness analysis (CEA) of malaria interventions can provide essential information for malaria control at various levels and can inform health sector budgets [3]. In this context, intervention is defined as any preventive, promotive, curative, or rehabilitative action that improves health [4]. CEA uses a cost-effectiveness ratio (CER) to compare interventions in terms of costs and effectiveness [5]. CEA can be used to identify priority interventions when resources are limited [6] and 
hence should be considered when designing strategies for prevention, treatment and control of disease [7]. CEA takes into account the costs and effects of adding new interventions to current ones or of replacing an existing intervention with another targeting the same condition [4]. The decision to employ a particular malaria intervention must therefore be determined, not only by the effectiveness of the intervention, but also by the ability of the health system to sustain its use [8].

The disability-adjusted life year (DALY) is a metric measure for burden of disease $[9,10]$ developed by the World Health Organization (WHO), World Bank and the Harvard School of Public Health researchers [1013]. DALYs were first used in the global burden of disease and injury (GBD) study, a joint study done by the World Bank, WHO and Harvard School of Public Health $[10,14]$. The DALY can be used as a summary measure to determine the cost-effectiveness of different types of interventions for each specific disease type [15]. It has been recommended that analysts express CER in terms of DALYs, although measures such as the quality-adjusted life years (QALYs) and healthy life years (HYL) can also be used [15]. CERs can be expressed as the cost per DALY averted through each intervention thereby allowing for comparisons in costs and effectiveness across different settings.

Information on CEA of malaria interventions is essential in informing malaria control programmes to guide the decision-making and planning processes. There is paucity of information on CEA of malaria interventions in most low- and medium-income countries, which are home to the majority of the impoverished communities of the world [15]. This may result in failure to effectively implement intervention programs at sufficient scale [5]. Hence, this review was conducted to assess and examine the utility of the DALY methodology in CEA of malaria interventions.

\section{Methods}

In this review, only studies employing the DALY methodology in CEA of malaria interventions were included. Selection for eligible studies was conducted through a search of peer-reviewed journals on Medline (PubMed) and EMBASE. Grey literature was also searched using the Google Scholar search engine. The searches included international peer-reviewed articles published in the period from 1996 (the year of the first GBD study $[10,14])$ to 1 June 2016 . The search terms were 'cost' OR 'effectiveness' OR 'cost-effectiveness' AND 'disability adjusted life years' AND 'malaria'. Any literature which did not satisfy these criteria was excluded. The snowball technique was used to identify other articles by searching for relevant papers listed in reference lists of the initially selected articles. Although review papers were not included as part of this review, they used to check for other potential references that fulfil the eligibility criteria. A total of 82 studies were initially retrieved and after further screening using the inclusion criteria, a total of 40 studies were finally included for this review. The inclusion criteria are shown by the PRISMA flow diagram (Fig. 1) which was adapted from Moher et al. [16] and modified. We limited our search to papers written in English. For each of the selected studies, we noted the year of study, malaria intervention assessed, intervention target population, country of study, data sources and CERs in terms of DALYs averted. We checked if the studies followed the GBD study methodology in estimating DALYs. We assessed the use of disability weights, application of age weighting and discounting as well as use of life expectancy tables.

\section{Cost-effectiveness analysis}

Reviewed studies used two main approaches as thresholds to determine whether or not an intervention was cost-effective. The first approach was based on per capita gross domestic product (GDP). Interventions with a CER per DALY averted less than a country's per capita gross domestic product (GDP) could be regarded as 'very cost-effective' and those for which the cost-effectiveness is less than three times the country's per capita GDP as 'cost-effective.' This approach was recommended by the World Health Organisation's choosing interventions that are cost-effective (WHO-CHOICE) project $[15,17]$. The second approach used was thresholds of US\$ 30 and US\$ 150 per DALY averted as a basis for considering an intervention either highly cost-effective or cost-effective respectively $[18,19]$.

In order to standardise the CERs, each ratio was expressed as number of DALYs averted per US\$ 1 million spent on the intervention. For CERs expressed as a range, the midpoint value was calculated and used calculate the number of DALYs averted.

\section{Results \\ Characteristics of reviewed studies}

Most of the reviewed studies $(\mathrm{n}=30)$ were done in Africa or used the African settings to model the CEA. The studies were published between 1999 and 2016. Figure 2 shows the number of reviewed studies against the year of publication. The highest number of studies were published in 2009 and 2014. The studies were done using data at different levels of coverage; district $(n=11)$, provincial $(\mathrm{n}=4)$, national $(\mathrm{n}=3)$ and regional $(\mathrm{n}=5)$ levels. Some studies $(n=17)$ did not specify the level of coverage as most of them used modelling to determine the cost-effectiveness of the interventions. Other studies 


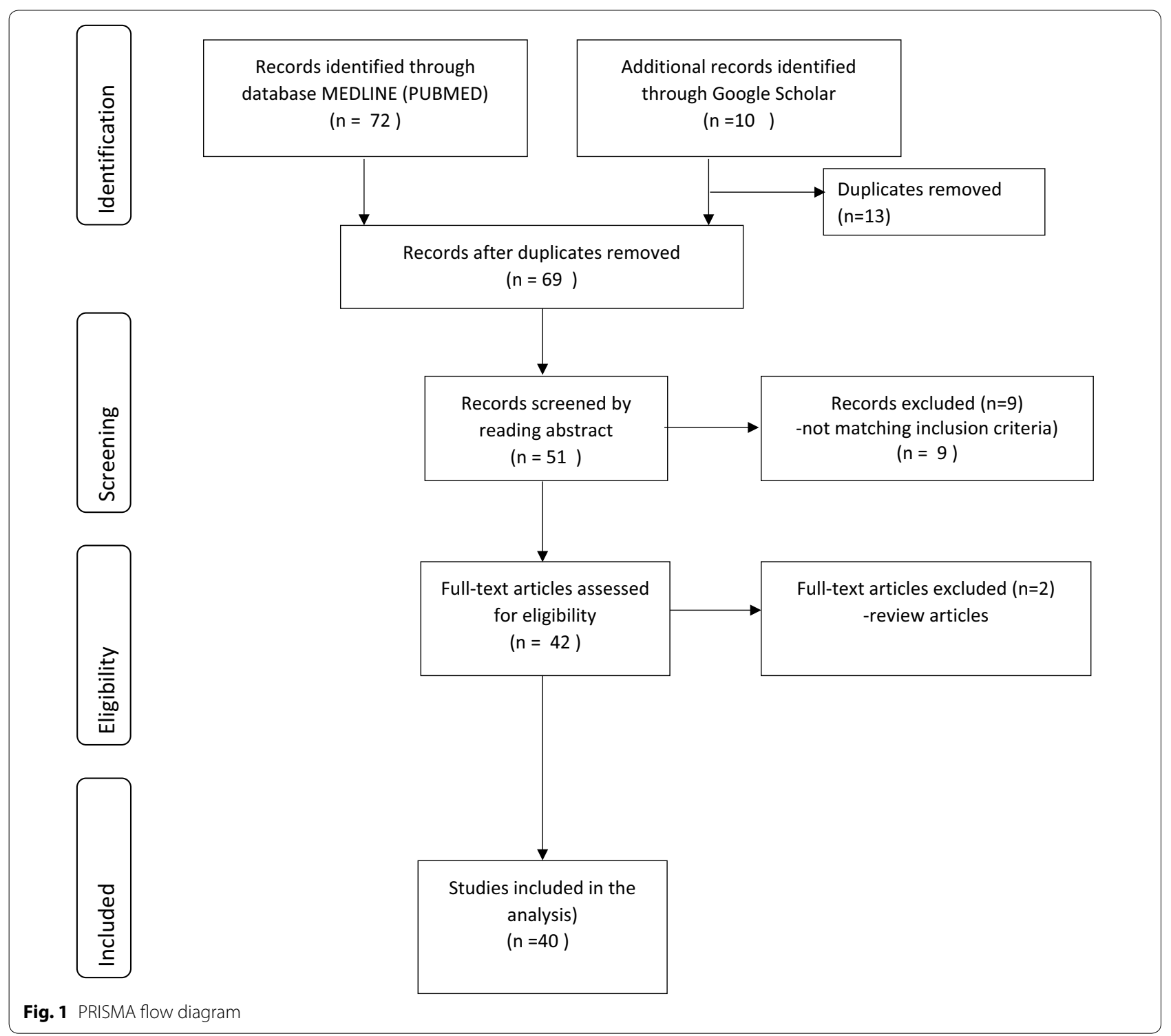

$(\mathrm{n}=21)$ stated the type of malaria (Plasmodium falciparum or Plasmodium vivax malaria) targeted by the intervention. Of the studies that stated the type of malaria, the majority of them were on interventions against $P$. falciparum. Sixty percent (60.0\%) of the malaria interventions targeted all age categories, while the others targeted pregnant women (12.5\%), children $(25.0 \%)$ and both women and children $(2.5 \%)$. Of the studies whose interventions targeted children, $20.0 \%(n=8)$ of them specifically targeted infants.

\section{DALY methodology used}

The DALY methodology was generally applied in a standard manner by most of the studies under review. However, some aspects of DALY calculation such disability weights and life expectancy values were not clearly stated in some instances. It was also not clear which specific assumptions were made when the calculation of DALYs was done. It was found that 19 studies (47.5\%) applied the same disability weight for malaria from the global burden of disease (GBD) studies. The first GBD study was published in 1996 [10] and the latest one in 2015 [20]. Other studies either applied country or region specific disability weights. However, 15 studies (37.5\%) did not clearly specify which disability weights for malaria were used to calculate the DALYs. Four (4) reviewed studies reported that they applied age weighting while six studies did not report on the use of age-weighting. Thirty-two (32) studies $(82.5 \%)$ reported applying a $3 \%$ discounting rate while six studies did not clearly specify whether discounting 


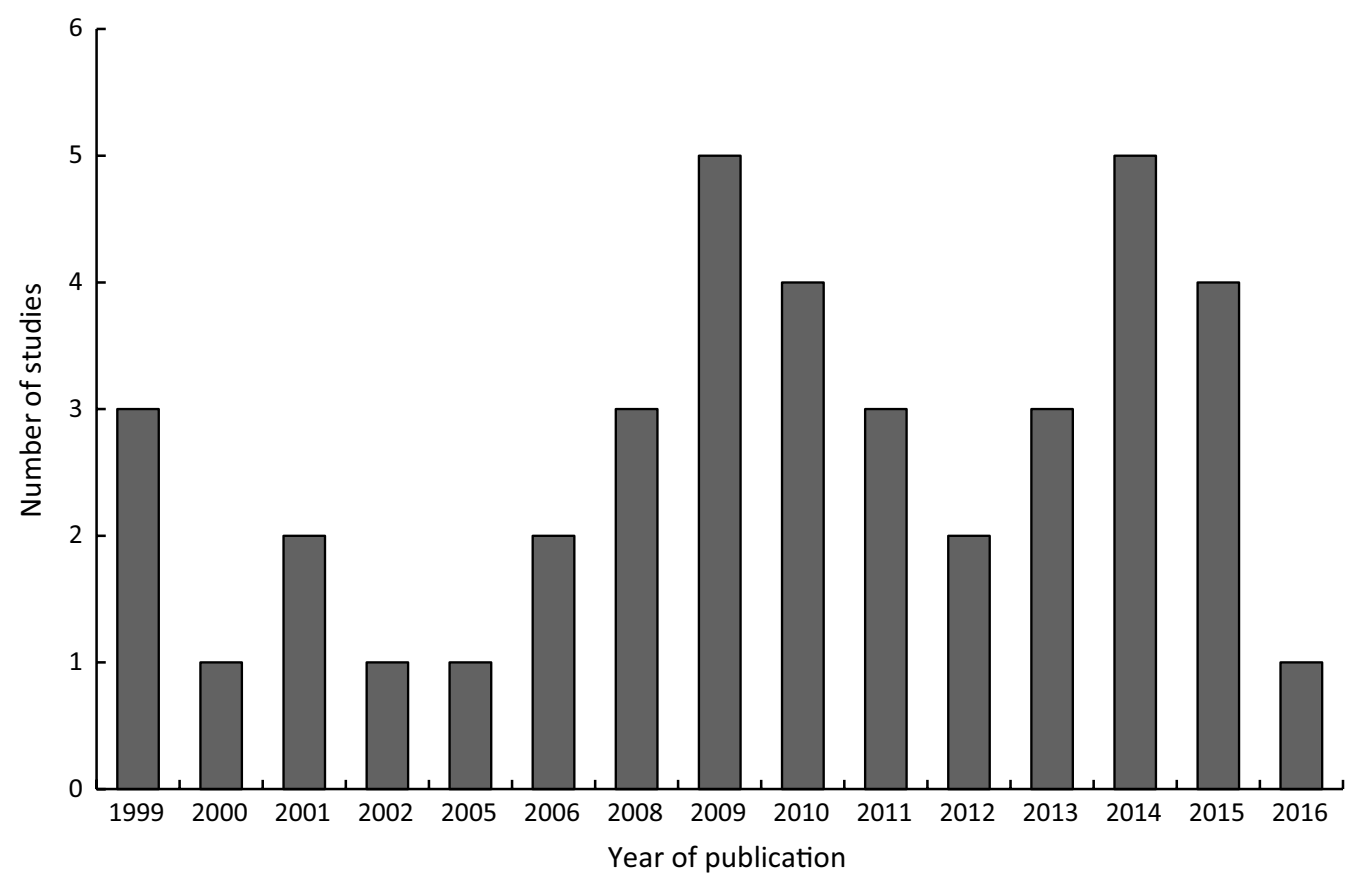

Fig. 2 Number of reviewed studies per year (1999-June 2016) that used DALYs in cost-effectiveness analysis

was applied or not. Twenty-seven studies (67.5\%) used country-specific life tables while $6(15.0 \%)$ used life tables obtained from the GBD study or WHO. Some of the studies 6 (15.0\%) did not explicitly state the source of the life expectancy used. The major sources of data used for calculating the DALYs included published and unpublished studies, malaria control program records, health facility records, clinical trials, programme reports and census data.

\section{Cost-effectiveness ratios}

In this review, we identified 40 studies on CEA of malaria interventions, which used DALYs to determine CERs in terms of cost per DALY averted per each intervention. The CERs were expressed in United States Dollars (US\$) per DALY averted and ranged from 1 US\$ to 639 US\$ depending with the specific intervention. Some studies expressed the CERs as cost of DALYs averted per person while in other studies it was expressed as the number of DALYs averted over a certain period of time. Other studies $(15.0 \%)$ did not specify the exact number of DALYs averted per each intervention. To standardise the CERs, we also expressed cost-effectiveness as DALYs averted per 1 million US\$ spent on each intervention. Based on internationally accepted thresholds for CERs [15, 17-19], most of the interventions (85\%) were found to be costeffective. For the malaria interventions that were targeted at all age groups (Table 1), pre-referral rectal antimalarial treatment and dihydroartemisinin-piperaquine (DP) combined with artemether-lumefantrine (AL) were the most cost-effective interventions. Each of these interventions averted approximately 200,000 DALYs per 1 million US\$ spent on the intervention. In some cases, the same intervention was more cost-effective in one setting compared to a different setting. For example, intermittent preventive treatment had a CER of US\$ 41.46 per DALY averted in Mozambique, while the same intervention had a CER of US\$ 136.30 when it was carried out as part of a multi-country study.

Combined interventions targeting pregnant women were the most cost-effective (Table 2). The combined interventions included provision of insecticide treated nets (ITNs), residual spraying, chemoprophylaxis and improved case management in pregnant women (IPTp) was the least cost-effective. The number of DALYs averted per 1 million US\$ spent on each intervention ranged from 19,231 to 222,222 DALYs.

Intermittent preventive treatment (IPT) in children (Table 3) in Mozambique and Tanzania was the most cost-effective intervention, averting 270,270 DALYs per 1 million US\$ spent on the intervention. Vaccines and long lasting insecticide-treated nets (LLITNs) were the least cost effective interventions for children and infants.

The least cost-effective intervention was rapid diagnostic tests (RDTs) in Myanmar, averting only 1565 DALYs per 1 million US\$ spent on the intervention. Although 


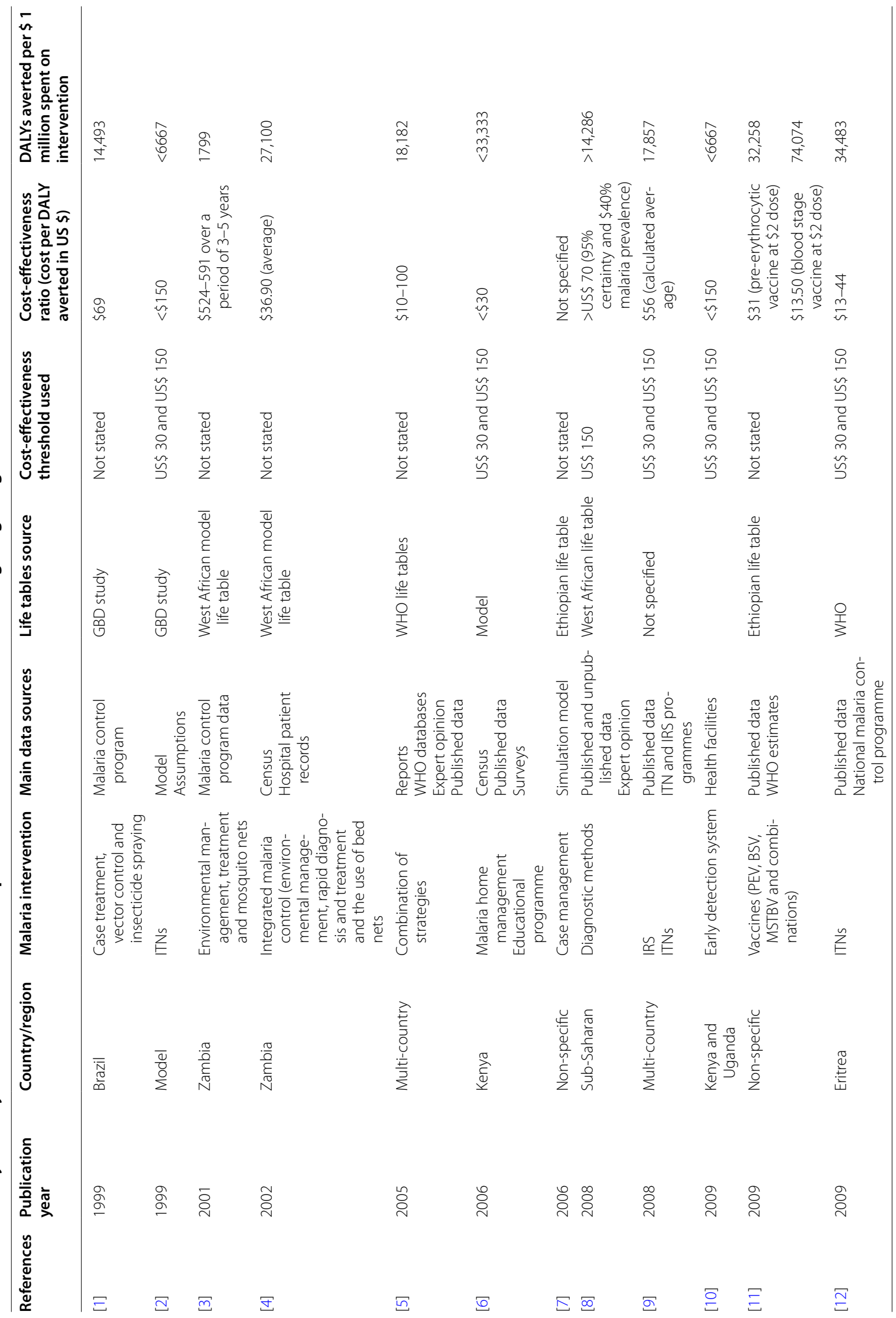




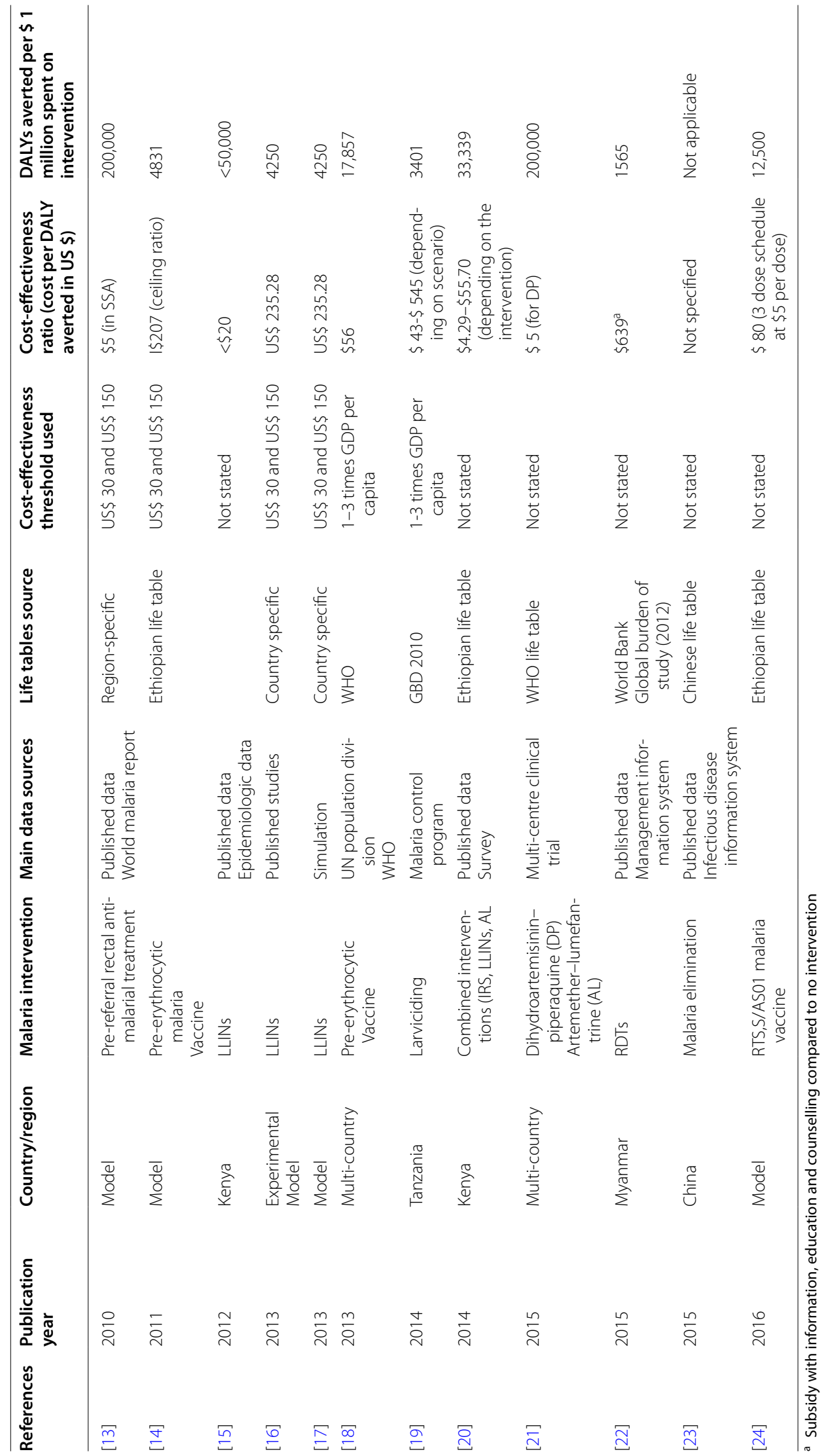




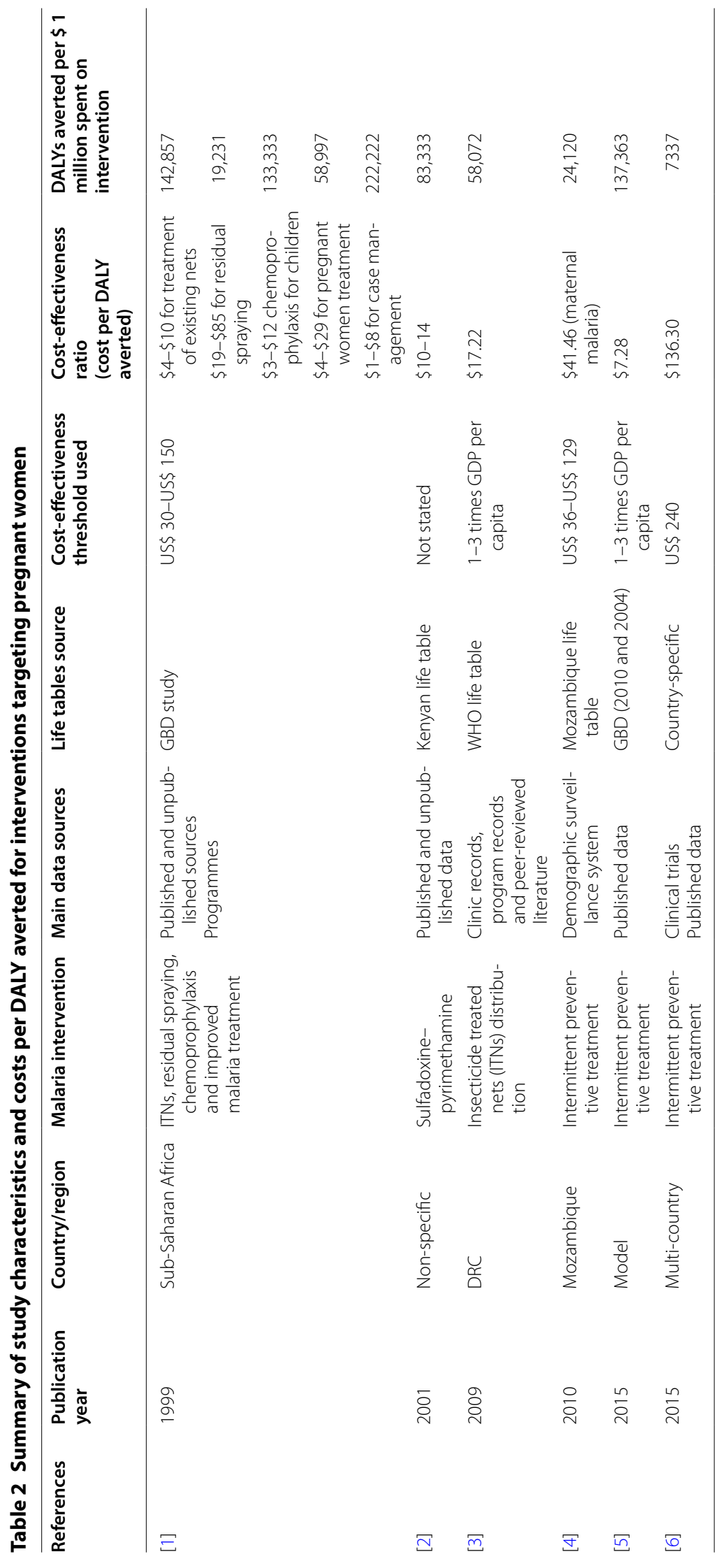




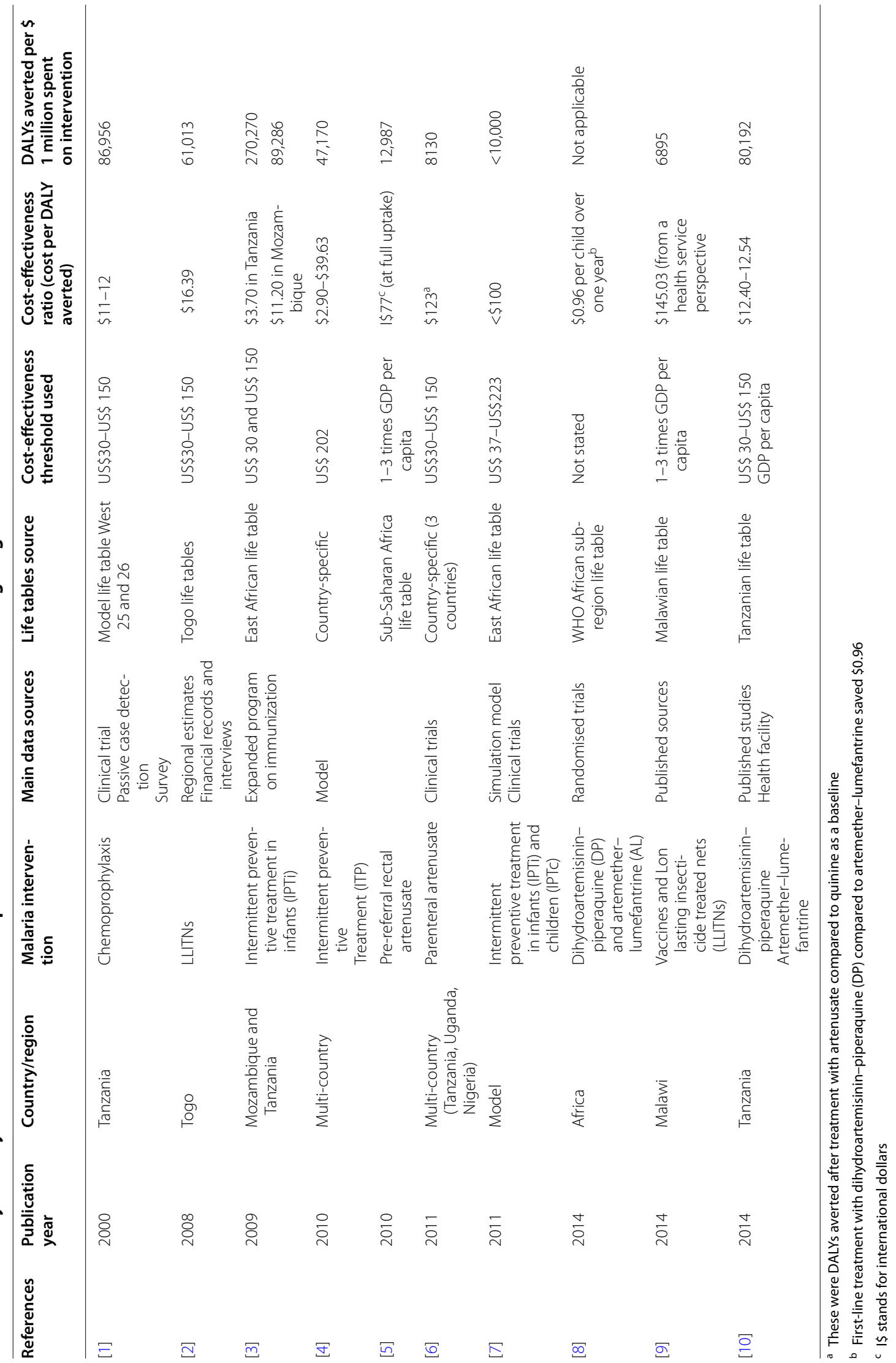


most interventions were cost-effective, some studies (35\%) did not specify the specific thresholds that were applied to determine cost-effectiveness.

\section{Malaria interventions}

Broad malaria intervention categories for the reviewed studies are summarised in Table 4. The interventions were classified as case treatment, prophylaxis, vector control, insecticide treated nets, early detection, environmental management, diagnosis, combined interventions and educational programmes. Most CEA studies were on case treatment (30.0\%) and preventive treatment (30.0\%) followed by insecticide treated bed-nets (27.5\%). However, some studies analysed cost-effectiveness of more than one malaria intervention.

\section{Case treatment}

Pre-referral artenusate was shown to be a cost-effective intervention in the management of severe childhood malaria in rural African settings with a cost of 77 international dollars (I\$) per DALY averted at full uptake [25]. Parenteral artenusate was highly cost effective and an affordable alternative to quinine for treating children with severe malaria with a cost of $\$ 123$ per DALY averted after treatment with artenusate compared to quinine as a baseline [8]. One study showed that combined rectal formulations (antimalarials and antibacterials) are a costeffective alternative to rectal anti-malarials or anti-bacterials alone [22]. This intervention showed a cost of $\$ 5$ per DALY averted. Three of the reviewed studies compared the cost-effectiveness of dihydroartemisinin-piperaquine (DP) and artemether-lumefantrine (AL) in treatment of complicated malaria $[21,23,55]$. The maximum cost per DALY averted for these three studies was $\$ 12.54$. These

\begin{tabular}{|c|c|c|}
\hline Intervention category & $\begin{array}{l}\text { Number } \\
\text { of studies (\%) }\end{array}$ & References \\
\hline $\begin{array}{l}\text { Case treatment (out- and } \\
\text { in-patients) }\end{array}$ & $12(30.0)$ & {$[3,8,21-30]$} \\
\hline Prophylaxis & $12(30.0)$ & {$[3,29,31-40]$} \\
\hline $\begin{array}{l}\text { Vector control/insecticide } \\
\text { spraying }\end{array}$ & $6(15.0)$ & {$[3,27,29,30,41,42]$} \\
\hline Insecticide treated nets & $11(27.5)$ & {$[3,28-30,40,42-49]$} \\
\hline Early detection system & $1(2.5)$ & {$[19]$} \\
\hline Environmental management & $2(5.0)$ & {$[28,50]$} \\
\hline Diagnosis & $2(5.0)$ & {$[51,52]$} \\
\hline Educational programme & $1(2.5)$ & [53] \\
\hline Malaria elimination program & $1(2.5)$ & {$[54]$} \\
\hline Combination of interventions & $6(15.0)$ & {$[3,27,28,30,40,50]$} \\
\hline
\end{tabular}

two drugs are highly recommended for the treatment of uncomplicated malaria. It was predicted that DP was more cost-effective compared to AL with the assumption that compliance to treatment was higher in DP than in $\mathrm{AL}$ due to the once a day dose for DP [23]. It was also suggested that DP might be more cost effective than AL across a range of settings in Africa [55].

\section{Prophylaxis}

Intermittent preventive treatment (IPT) is one of the malaria control strategies used in malaria endemic areas. This strategy is often used in infants and pregnant women and can contribute to a decrease in clinical malaria [32]. IPT in infants (IPTi) was shown to be a highly costeffective intervention when it is delivered alongside the expanded programme on immunisation (EPI) with a range of $\$ 2.90-\$ 29.63$ per DALY averted [32]. This strategy can be strengthened by inclusion of iron supplementation [33]. IPTi with sulfadoxine pyrimethamine (SP) is expected to produce health improvements in a costeffective way from both the health system and societal perspectives [31]. One study demonstrated the importance of CEA for IPT in pregnant women (IPTp) in supporting policymakers' decisions [56]. The study showed that monthly doses of SP during the second and third trimester are more cost effective than only two doses that were previously recommended. This finding was consistent with the WHO guidelines [57].

One reviewed study estimated the cost-effectiveness of IPTp-SP on maternal clinical malaria and neonatal survival in Mozambique [38] and found that it was costeffective in both instances. The cost per DALY averted for maternal malaria was $\$ 41.46$. This intervention was said to remain cost-effective even with significant increases in drug and other related costs. Presumptive treatment regimens to prevent low birth weight associated with malaria were shown to be a cost-effective strategy in areas with high malaria transmission rates [26]. The cost-effectiveness of 2-dose IPTp-mefloquine (MQ) was compared with IPTp-SP in HIV negative women. IPTp-MQ was more cost-effective than IPTp-SP although poor tolerability of MQ does not favour its use for IPTp [58].

A malaria vaccine model was applied to analyse the cost-effectiveness of a hypothetical pre-erythrocytic malaria vaccine [34]. Using a ceiling ratio of I $\$ 207$ as cost per DALY averted, this study showed that $52.4 \%$ of parameterizations predicted cost-effectiveness in the primary analysis. The cost-effectiveness of the vaccine was shown to be maximal in low endemicity settings, thereby suggesting the use of a selective vaccine introduction strategy. Vaccinating children with RTS,S vaccine was shown to be very cost-effective from both a societal and health service perspective in Malawi [40]. However, the 
study recommended that long-term follow-up studies were essential. The RTS,S/AS01 vaccine was shown to be highly cost-effective across a wide range of African settings [36].

Simulation showed that malaria vaccines might be an efficient malaria control intervention and that both transmission setting and vaccine delivery modality are important to their cost-effectiveness [39]. The simulation used three different vaccine types: pre-erythrocytic vaccines (PEV), blood stage vaccines (BSV) and mosquitostage transmission-blocking vaccines (MSTBV). The cost per DALY averted for PEV and BSV was $\$ 31$ and $\$ 13.50$ respectively at a cost of $\$ 2$ per dose. Specific malaria intervention drugs used in prophylaxis, case treatment and vaccines are summarised in Table 5 . SP was the most common drug of choice in malaria prophylaxis, while artemisinin-based combination therapies (ACTs) were the most common drugs used in case treatment. In addition to prophylaxis, SP was also used for case treatment.

\section{Insecticide treated nets and vector control}

The effectiveness of ITNs in preventing malaria is threatened by increasing resistance to insecticides as well as changing biting behaviour of mosquitoes $[44,45]$. Combination mosquito nets such as pyrethroid piperonyl butoxide long lasting insecticidal nets were shown to be likely more cost effective than standard long lasting insecticidal nets (LLINs), especially in areas with strong resistance to pyrethroids [44]. Varying malaria transmission levels were shown to have an impact on CERs.

There is paucity of information on the cost-effectiveness of larviciding. In light of this, a study was carried out in Tanzania to estimate the CERs of microbial larviciding for malaria vectors [41]. The study estimated CERs from the provider and societal perspectives, and showed that larviciding can be used as a cost-effective intervention in urban areas with the cost per DALY averted in the range $\$ 43-\$ 545$. A study on the cost-effectiveness of a malaria control program showed case treatment to be more costeffective than vector control, in particular, in areas where $P$. falciparum is prevalent and insecticide spraying is relatively ineffective [27]. The cost per DALY averted was shown to be $\$ 69$.

The cost-effectiveness of elimination of falciparum malaria was analysed in a province in China [54], with the results of the study showing that the programme was cost-effective. The cost-effectiveness of an early detection system for epidemic malaria was explored in the highlands of Kenya and Uganda [19]. Results from the study suggested that the early detection system was cost-effective, but further studies are needed to analyse the costs and effects of the health systems' reaction after being prompted by the early detection system.

There is scanty information on the cost-effectiveness of environmental management as a malaria control strategy. Hence, a study was conducted to assess the efficacy and cost-effectiveness of environmental management (vegetation clearance, modification of river boundaries, draining swamps, oil application to open water bodies and house screening) [28]. The results of that study showed that environmental management, when integrated with other malaria control interventions like case treatment, insecticide spraying and bed nets, could substantially increase the chances of rolling-back malaria. A study in rural Kenya found that an educational programme for home management of malaria targeted at shopkeepers and communities was highly cost-effective when compared to other benchmarks for interventions in resource-limited settings [53]. The strategy of introducing an education programme is therefore essential in areas where a large proportion of the community access malaria drugs from private retailers.

\section{Diagnosis}

A subsidy of RDTs and artemisinin-based combination therapies (ACTs) within the informal private sector can help in the efforts to fight malaria. When these subsidies are combined with information, education and counselling, the results were shown to have favourable CERs [51]. RDTs were shown to have the potential to be cost-effective in most parts of sub-Saharan Africa. This reflected better treatment and health outcomes for

Table 5 Specific malaria intervention drugs used for prophylaxis, case treatment and vaccines

\begin{tabular}{llllll}
\hline Prophylaxis & References & Case treatment & References & Vaccine & References \\
\hline Sulfadoxine-pyrimethamine & {$[26,29,31,32,37,38,56,58]$} & Dihydroartemisinin-piperaquine (DP) & {$[21,23,29,55]$} & RTS,S & {$[34]$} \\
Mefloquine & {$[32,58]$} & Artemether-lumefantrine (AL) & {$[21-23,29,30,55]$} & Hypothetical & {$[35]$} \\
Chlorproguanil-dapsone & {$[32]$} & Artenusate & {$[8,22,25]$} & RTS,S/AS01 & {$[36]$} \\
Artenusate & {$[32]$} & Quinine & {$[22]$} & & \\
Amodiaquine-artenusate & {$[32,37]$} & Sulfadoxine-pyrimethamine & {$[3,24,29]$} & \\
Pyrimethamine-dapsone & {$[33]$} & Amodiaquine & {$[24]$} & & \\
Chloroquine & {$[3]$} & Chloroquine & {$[29]$} & & \\
\hline
\end{tabular}


non-malarial febrile illness as well as savings on antimalarial drug costs [52].

\section{Combination of interventions}

A stochastic simulation modelling platform was applied to simulate the impact of interventions singly and in combination in the highlands of Kenya [30]. The study results showed that the greatest simulated health impact was from a combination of long-lasting insecticidal nets (LLIN) use by $80 \%$ of the population, $90 \%$ household coverage by IRS with deployment starting in April and intermittent screen and test of school children using $\mathrm{AL}$ with $80.0 \%$ coverage twice per term. It was also shown that high coverage with artemisinin-based combination treatments is the most cost-effective strategy in most countries in sub-Saharan Africa with the cost per DALY averted in the range \$10-100 [29]. However, this alone is not enough if it is not combined with other interventions such as use of ITNs, IRS and ITP. The economic impact of malaria was assessed in the mining sector in the Zambian copperbelt. The study showed that integrated malaria control in the copper mining communities was a sound investment resulting in reduced direct malaria treatment costs and reduced indirect costs as a result of reduced work absenteeism [50].

\section{Discussion}

We reviewed studies that utilised the DALY metric in cost-effectiveness analysis of malaria interventions. Although the reviewed studies used the DALY, there were some variations in methodology. Most of the interventions were within the WHO-CHOICE thresholds for cost-effectiveness. Some interventions were more costeffective in one setting compared to a different setting. This shows that cost-effectiveness analysis may only be useful in the context of the choices available in a particular setting [59]. Although most interventions reviewed in this study were cost-effective based on set thresholds, the number of DALYs averted per one million US\$ spent on each intervention were different. It is therefore essential for policymakers to compare results of cost-effectiveness analysis with as many relevant interventions as possible before making resource allocation decisions.

In general, a combination of interventions were more cost-effective than single interventions. Combined malaria interventions have been shown to deliver substantial efficiency gains compared to single interventions [48]. For instance, ITN distribution was shown to be a more cost-effective intervention when added to antenatal services [43]. It is essential to include long-term surveillance as part of ITN interventions, with particular attention to the age range over which rebound can occur [46]. It has been shown that emphasis on treatment as well as targeted vector control yielded significantly lower costs per life saved [27]. Although there is a wide range of costeffective interventions available, provision of these intervention packages is often not affordable in low-income countries. There is therefore, need for external donors to assist with funding where possible [3]. Information from the studies in this review can be used to make decisions on which interventions can be effectively applied independently and which ones are mutually exclusive [4].

The majority of published studies on CEA of malaria interventions using DALYs focused on case treatment, use of insecticide treated nets and prophylaxis. This review showed that there is paucity of information on cost-effectiveness of other interventions such as early detection, environmental management, diagnostic services and educational programmes. Lack of cost-effectiveness information on some interventions makes it difficult to conduct a comprehensive comparison in order to guide policy-makers in decision-making. There is therefore need for more CEA studies on less explored malaria interventions to inform policy and to improve effectiveness of these interventions. The evidence provided by such studies will assist in guiding decisions at various levels [6]. From our literature search, there were no studies on cost-effectiveness of malaria interventions for a malaria outbreak scenario. It would be interesting to know whether interventions that are likely to be costeffective in a normal malaria transmission situation will also be cost-effective in an outbreak.

This review showed that disease modelling methods can provide useful information by predicting cost-effectiveness for scenarios and multiple strategies, where, for practical reasons, trials cannot be carried out [3, 37]. However, CEA results obtained through modelling techniques must be interpreted with caution as assumptions made in the models may be different to the actual situation obtaining in real life situations. Thus, results from complex models should be presented to decisionmakers in a form in which interpretation and translation is easy.

Comparison of CERs among the reviewed studies was difficult as the number of DALYs averted per each intervention were often expressed differently. Although most studies expressed CERs as cost per DALYs averted, some studies only gave a range without specifying the mean number of DALYs averted. Some of the studies looking at a combination of interventions did not give a breakdown of the cost per DALY averted for each individual intervention. In some cases, there was very little information on the methodological choices made. For example, some studies did not specify the disability weight which was used, the sources of data on malaria incidence and the source of life expectancy values. In some cases, this 
information had to be extracted from referenced articles as it was not clearly stated.

\section{Conclusions}

Cost-effectiveness analysis studies of malaria interventions done over the past 20 or so years have provided important information for policymakers to guide them on choosing the most cost-effective interventions for malaria programmes. This review has shown that most malaria interventions are cost-effective in terms of the cost per DALYs averted per each malaria intervention, based on acceptable thresholds. This information is useful in identifying interventions that effectively use available resources. Although most of the studies we reviewed generally followed the DALY methodology in the CEA, there were differences in the way the CERs were expressed thereby making it difficult to make a comprehensive comparison between studies.

\begin{abstract}
Abbreviations
AL: artemether-lumefantrine; CEA: cost-effectiveness analysis; CER: cost-effectiveness ratio; DP: dihydroartemisinin-piperaquine; DALY: disability adjusted life year; GBD: global burden of disease; GDP: gross domestic product; ITP: intermittent preventive treatment; ITNs: insecticidal treated nets; RDT: rapid diagnostic test; US\$: United States Dollars; WHO: World Health Organisation.
\end{abstract}

\section{Authors' contributions}

RG led on all aspects of the paper's development from conceptualisation to finalisation, while MJC was involved in the conceptualisation and reviewing of all drafts of the paper. Both authors read and approved the final manuscript.

\section{Acknowledgements}

Not applicable.

\section{Competing interests}

The authors declare that they have no competing interests.

\section{Availability of data and materials}

Data and materials can be requested from the authors.

\section{Funding}

No direct funding was received to support the reported research.

\section{Publisher's Note}

Springer Nature remains neutral with regard to jurisdictional claims in published maps and institutional affiliations.

Received: 13 January 2017 Accepted: 24 June 2017

Published online: 01 July 2017

\section{References}

1. WHO. World malaria report. Geneva: WHO; 2015.

2. Githeko AK, Ndegwa W. Predicting malaria epidemics in the Kenyan highlands using climate data: a tool for decision makers. Glob Chang Hum Health. 2001;2(1):54-63.

3. Goodman CA, Coleman PG, Mills AJ. Cost-effectiveness of malaria control in sub-Saharan Africa. Lancet. 1999;354(9176):378-85.

4. Evans DB, et al. Methods to assess the costs and health effects of interventions for improving health in developing countries. BMJ. 2005;331(7525):1137-40.
5. Galarraga O, et al. HIV prevention cost-effectiveness: a systematic review. BMC Public Health. 2009;9(Suppl 1):S5

6. Chisin R. Cost-effectiveness analysis. J Nucl Med. 2009;50(3):338-9.

7. Creese A, et al. Cost-effectiveness of HIV/AIDS interventions in Africa: a systematic review of the evidence. Lancet. 2002;359(9318):1635-43.

8. Lubell $Y$, et al. Cost-effectiveness of parenteral artesunate for treating children with severe malaria in sub-Saharan Africa. Bull World Health Organ. 2011;89(7):504-12.

9. Bener $\mathrm{A}$, et al. Measuring burden of diseases in a rapidly developing economy: state of Qatar. Glob J Health Sci. 2013;5(2):134-44.

10. Murray CJL, Lopez A. The global burden of disease: a comprehensive assessment of mortality and disability from diseases, injuries, and risk factors in 1990 and projected to 2020. 1st ed. Cambridge: Harvard University Press; 1996.

11. Murray CJ, Lopez AD, editors. The Global Burden of Disease. Havard School of Public Health: Geneva; 1998. p. 1-46.

12. Donev D, Zaletel-Kragelj L, Bjegovic V, Burazeri G. Measuring the burden of disease: disability adjusted life year (daly). Methods and tools in public health. In: Zaletel-Kragelj L, Boţikov J, editors. A handbook for teachers, researchers and health professionals. Lage: Hans Jacobs Publishing Company; 2010.

13. Murray CJ, et al. GBD 2010: design, definitions, and metrics. Lancet. 2012;380(9859):2063-6

14. Murray CJL, Lopez AD. Global health statistics: a compendium of incidence, prevalence and mortality estimates for over 200 conditions. 2nd ed. Cambridge: Harvard University Press; 1996.

15. WHO. Making choices in health: WHO guide to cost-effectiveness analysis. In: Tan-Torres Edejer T, Baltussen R, Adam T, Hutubessy R, Acharya A, Evans DB, Murray DB, Murray CJL, editors. Geneva:WHO; 2003.

16. Moher $D$, et al. Preferred reporting items for systematic reviews and meta-analyses: the PRISMA statement. Open Med. 2009;3(3):e123-30.

17. Edwards C. Cost-effectiveness analysis in practice. In: Hunter P, Cameron J, Jagals P, Pond K, editors. Valuing water, valuing livelihoods. London: IWA Publishing; 2011. p. 181-197.

18. WHO. Investing in health research and development: report of the ad hoc committee on health research relating to future intervention options. Geneve: WHO; 1996.

19. Mueller $\mathrm{DH}$, et al. Costs of early detection systems for epidemic malaria in highland areas of Kenya and Uganda. Malar J. 2009;8:17.

20. GBD 2013 DALYs and HALE Collaborators. Global, regional, and national disability-adjusted life years (DALYs) for 306 diseases and injuries and healthy life expectancy (HALE) for 188 countries, 1990-2013: quantifying the epidemiological transition. Lancet. 2015;386(10009):2145-91.

21. Pfeil J, Borrmann S, Tozan Y. Dihydroartemisinin-piperaquine vs. artemether-lumefantrine for first-line treatment of uncomplicated malaria in African children: a cost-effectiveness analysis. PLOS ONE. 2014;9(4):e95681.

22. Buchanan J, et al. Cost-effectiveness of pre-referral antimalarial, antibacterial, and combined rectal formulations for severe febrile illness. PLOS ONE. 2010:5(12):e14446.

23. Mori AT, et al. Cost-effectiveness of dihydroartemisinin-piperaquine compared with artemether-lumefantrine for treating uncomplicated malaria in children at a district hospital in Tanzania. Malar J. 2014;13:363.

24. Tediosi F, et al. An approach to model the costs and effects of case management of Plasmodium falciparum malaria in Sub-Saharan Africa. Am J Trop Med Hyg. 2006;75(2 Suppl):90-103.

25. Tozan $Y$, et al. Prereferral rectal artesunate for treatment of severe childhood malaria: a cost-effectiveness analysis. Lancet. 2010;376(9756):1910-5

26. Wolfe EB, et al. Cost-effectiveness of sulfadoxine-pyrimethamine for the prevention of malaria-associated low birth weight. Am J Trop Med Hyg. 2001;64(3-4):178-86.

27. Akhavan D, et al. Cost-effective malaria control in Brazil: cost-effectiveness of a malaria control program in the Amazon Basin of Brazil, 1988-1996. Soc Sci Med. 1999:49(10):1385-99.

28. Utzinger J, Tozan $Y$, Singer BH. Efficacy and cost-effectiveness of environmental management for malaria control. Trop Med Int Health. 2001;6(9):677-87

29. Morel CM, Lauer JA, Evans DB. Cost effectiveness analysis of strategies to combat malaria in developing countries. BMJ. 2005;331(7528):1299. 
30. Stuckey EM, et al. Modeling the cost effectiveness of malaria control interventions in the highlands of western Kenya. PLOS ONE. 2014;9(10):e107700.

31. Hutton G. Cost-effectiveness of malaria intermittent preventive treatment in infants (IPTi) in Mozambique and the United Republic of Tanzania. Bull World Health Organ. 2009;87(2):123-9

32. Conteh $L$, et al. The cost-effectiveness of intermittent preventive treatment for malaria in infants in Sub-Saharan Africa. PLOS ONE. 2010;5(6):e10313.

33. Alonzo Gonzalez M, et al. Cost-effectiveness of iron supplementation and malaria chemoprophylaxis in the prevention of anaemia and malaria among Tanzanian infants. Bull World Health Organ. 2000;78(1):97-107.

34. Maire $\mathrm{N}$, et al. Cost-effectiveness of the introduction of a pre-erythrocytic malaria vaccine into the expanded program on immunization in subSaharan Africa: analysis of uncertainties using a stochastic individualbased simulation model of Plasmodium falciparum malaria. Value Health. 2011;14(8):1028-38.

35. Nunes JK, et al. Modeling the public health impact of malaria vaccines for developers and policymakers. BMC Infect Dis. 2013;13:295.

36. Penny MA, et al. Public health impact and cost-effectiveness of the RTS, S/AS01 malaria vaccine: a systematic comparison of predictions from four mathematical models. Lancet. 2016:387(10016):367-75.

37. Ross A, et al. Determinants of the cost-effectiveness of intermittent preventive treatment for malaria in infants and children. PLOS ONE. 2011;6(4):e18391.

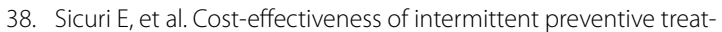
ment of malaria in pregnancy in southern Mozambique. PLoS ONE. 2010;5(10):e13407.

39. Tediosi F, et al. Simulation of the cost-effectiveness of malaria vaccines. Malar J. 2009;8:127

40. Seo MK, Baker P, Ngo KN. Cost-effectiveness analysis of vaccinating children in Malawi with RTS,S vaccines in comparison with long-lasting insecticide-treated nets. Malar J. 2014:13:66.

41. Maheu-Giroux M, Castro MC. Cost-effectiveness of larviciding for urban malaria control in Tanzania. Malar J. 2014;13:477.

42. Yukich $\mathrm{JO}$, et al. Costs and consequences of large-scale vector control for malaria. Malar J. 2008:7:258.

43. Becker-Dreps SI, et al. Cost-effectiveness of adding bed net distribution for malaria prevention to antenatal services in Kinshasa, Democratic Republic of the Congo. Am J Trop Med Hyg. 2009;81 (3):496-502.

44. Briet OJ, Chitnis N. Effects of changing mosquito host searching behaviour on the cost effectiveness of a mass distribution of long-lasting, insecticidal nets: a modelling study. Malar J. 2013;12:215.

45. Briet OJ, et al. Effects of pyrethroid resistance on the cost effectiveness of a mass distribution of long-lasting insecticidal nets: a modelling study. Malar J. 2013:12:77.
46. Coleman PG, Goodman CA, Mills A. Rebound mortality and the costeffectiveness of malaria control: potential impact of increased mortality in late childhood following the introduction of insecticide treated nets. Trop Med Int Health. 1999;4(3):175-86.

47. Kahn JG, et al. Integrated HIV testing, malaria, and diarrhea prevention campaign in Kenya: modeled health impact and cost-effectiveness. PLoS ONE. 2012;7(2):e31316.

48. Mueller DH, et al. Cost-effectiveness analysis of insecticide-treated net distribution as part of the Togo Integrated Child Health Campaign. Malar J. 2008; $7: 73$

49. Yukich JO, et al. Costs and cost-effectiveness of vector control in Eritrea using insecticide-treated bed nets. Malar J. 2009;8:51.

50. Utzinger J, et al. The economic payoffs of integrated malaria control in the Zambian copperbelt between 1930 and 1950. Trop Med Int Health. 2002;7(8):657-77.

51. Chen IT, et al. Cost-effectiveness analysis of malaria rapid diagnostic test incentive schemes for informal private healthcare providers in Myanmar. Malar J. 2015;14:55.

52. Shillcutt $\mathrm{S}$, et al. Cost-effectiveness of malaria diagnostic methods in subSaharan Africa in an era of combination therapy. Bull World Health Organ. 2008;86(2):101-10.

53. Goodman CA, et al. The cost-effectiveness of improving malaria home management: shopkeeper training in rural Kenya. Health Policy Plan. 2006;21(4):275-88

54. Sun DW, et al. A cost-effectiveness analysis of Plasmodium falciparum malaria elimination in Hainan Province, 2002-2012. Am J Trop Med Hyg. 2015;93(6):1240-8

55. Pfeil J, et al. An economic evaluation of the posttreatment prophylactic effect of dihydroartemisinin-piperaquine versus artemether-lumefantrine for first-line treatment of Plasmodium falciparum malaria across different transmission settings in Africa. Am J Trop Med Hyg. 2015;93(5):961-6.

56. Fernandes $\mathrm{S}$, et al. Cost-effectiveness of two versus three or more doses of intermittent preventive treatment for malaria during pregnancy in sub-Saharan Africa: a modelling study of meta-analysis and cost data. Lancet Glob Health. 2015;3(3):e143-53.

57. WHO. Intermittent preventive treatment in pregnancy (IPTp): key documents and guidelines. 2016. http://www.who.int/malaria/areas/ preventive_therapies/pregnancy/en/.

58. Sicuri E, et al. Economic evaluation of an alternative drug to sulfadoxine-pyrimethamine as intermittent preventive treatment of malaria in pregnancy. PLoS ONE. 2015;10(4):e0125072.

59. Marseille $\mathrm{E}$, et al. Thresholds for the cost-effectiveness of interventions: alternative approaches. Bull World Health Organ. 2015;93(2):118-24.

\section{Submit your next manuscript to BioMed Central and we will help you at every step:}

- We accept pre-submission inquiries

- Our selector tool helps you to find the most relevant journal

- We provide round the clock customer support

- Convenient online submission

- Thorough peer review

- Inclusion in PubMed and all major indexing services

- Maximum visibility for your research

Submit your manuscript at www.biomedcentral com/submit 